\title{
Combined Inhibitory Effect of Sulindac an NSAID and Quercetin an Antioxidant on Bleomycin-induced Lung Fibrosis in Rats
}

\section{Ramesh Verma ${ }^{1}$, Poonam Mehta ${ }^{1}$, Vishvesh Dalal ${ }^{1}$, Manish V Patel ${ }^{1}$ and Suresh Balakrishnan ${ }^{2 *}$}

${ }^{1}$ Department of Toxicology, Jai Research Foundation, Gujarat, India

${ }^{2}$ Division of Toxicology, Department of Zoology, Faculty of Science, The M.S.

University of Baroda, Vadodara, Gujarat, India

*Corresponding Author: Suresh Balakrishnan, Division of Toxicology, Department of Zoology, Faculty of Science, The M.S. University of Baroda, Vadodara, Gujarat, India.
Received: June 23, 2021

Published: August 18, 2021

(C) All rights are reserved by Suresh

Balakrishnan., et al.

\begin{abstract}
Idiopathic pulmonary fibrosis is the most prevalent chronic lung disease. Studies have suggested that ROS such as superoxide anion, hydrogen peroxide and hydroxyl radical are major mediators of lung inflammatory processes. So, the study was conducted to evaluate the ameliorative effects of quercetin alone and in combination with sulindac on lung injury induced by bleomycin. Oral administration of quercetin (100 mg/kg/day) or sulindac (20 mg/kg/day) for 20 days, attenuated the severity of bleomycin induced lung injury and fibrosis through decreasing the adverse effect of bleomycin on lung hydroxyproline contents and plasma TNF- $\alpha$ levels alike. However, subtle signs of toxicity still prevailed. Nevertheless, it was noticed that combined low doses of quercetin (50 mg/kg/ day) and sulindac (10 mg/kg/day) provided more benefits than full separate doses of each compound on the deleterious effects accompanied bleomycin administration. Therefore, it is pertinent to presume that concurrent administration of quercetin and sulindac has synergistic effect.
\end{abstract}

Keywords: Idiopathic Pulmonary Fibrosis; Bleomycin; Quercetin; Sulindac; Hydroxyproline

\section{Introduction}

Pulmonary fibrosis is interstitial lung disease with fatal prognosis and poor response to available medical therapy, characterized by excessive deposition of extracellular matrix in the airspace thought to be initiated by acute or chronic lung injury $[13,15,35]$. Although the pathophysiology of pulmonary fibrosis has yet to be understood, it has generally been hypothesized that activated inflammatory cells which accumulate in the lower airways, release harmful amount of reactive oxygen species which result in lung injury and proliferation of fibroblast in alveolar walls [51]. The activated fibroblasts produce excessive amount of extracellular matrix proteins that impair the vital gas exchange function of the lungs [9]. Inflammation and immune processes are, however, considered among the major mechanisms that injure lung tissue and induce fibrosis [16]. There is also considerable evidence that oxidative stress due to oxygen generated free radicals plays a major role in inflammatory and immune-mediated bleomycin-induced lung damage $[31,42]$.

A variety of animal models of pulmonary fibrosis have been developed to examine potential therapies for idiopathic pulmonary fibrosis. Bleomycin, a chemotherapeutic antibiotic drug which induces pulmonary parenchymal fibrosis in rats is the most established experimental model of pulmonary fibrosis [26].

The bleomycin induces the genesis of reactive oxygen species upon binding to DNA and iron, which in turn causes DNA damage [1]. The interaction of bleomycin with DNA is postulated to initiate the inflammatory changes through a concerted action of vari- 
ous cytokines leading to excessive accumulation of collagen in the lung tissue [6,50]. Further, it is reported that bleomycin promotes the depletion of endogenous antioxidant defences thus exacerbating oxidant mediated tissue injury [1]. The lung is selectively affected by bleomycin because it lacks an enzyme that hydrolyzes the $\beta$-aminoalanine moiety of bleomycin, which prevents its metabolite from binding to metals such as iron $[2,10,12,38,40]$.

Quercetin (3,3目,4目,5,7-pentahydroxyflavone), a flavanoids present in fruits and vegetables has attracted much attention for its beneficial health effects due to its multiple mechanisms including antioxidant activity, anti-inflammation, modification of signal transduction pathways and interactions with receptors and other proteins. The antioxidant activity of quercetin is primarily credited to its phenolic hydroxyl groups [25]. Sulindac ([Z]-5-fluoro2-methyl-1-[p-(methylsulfinyl)-benzylidene]indene-3-acetic acid), a non-steroidal anti-inflammatory drug, is well known for its antiinflammatory activity, which is due to its ability to inhibit the cyclooxygenases enzymes and thereby inhibits prostaglandin synthesis $[36,46]$.

Therefore, the present study was conducted to elucidate the possible inhibitory effects of quercetin on bleomycin induced rat pulmonary fibrosis compared to that afforded by sulindac. As observed in many multifaceted disorders, it is likely that grouping of agents, rather than single treatments will be needed. According to the best of our knowledge, no data dealt with the combined inhibitory effects of quercetin and sulindac. Therefore, the present study was extended to evaluate the inhibitory effects of quercetin in combination with sulindac on bleomycin induced rat pulmonary fibrosis as quercetin possess an antioxidant while sulindac is well known for its anti-inflammatory activity.

\section{Materials and Methods}

Animals

Specific pathogen-free, healthy young adult male Wistar rats (RccHan:WIST), of 10 to 12 weeks were used in this study. They were obtained from the Barrier Maintained Rodent Animal Breeding Facility, Jai Research Foundation, Vapi, India. All the animals were fed with Teklad Certified Global rat feed manufactured by Harlan, U.S.A. and U.V. sterilised water filtered through Reverse Osmosis water filtration system was provided, ad libitum. The rats were kept in a controlled environment (temperature: $22 \pm 3^{\circ} \mathrm{C}$ and relative humidity: 30 to $70 \%$ ) with an alternating cycle of 12-h light and dark. The animals used in this study were handled and treated in accordance with the strict guiding principles of the National Institutes of Health Guide for the Care and Use of Laboratory Animals and Association for Assessment and Accreditation of Laboratory Animal Care (AAALAC). The test facility at Jai Research Foundation is AAALAC accredited and is also complies with the National Good Laboratory Practice, India. The experimental protocols were approved by the Institutional Animal Ethics Committee (Approval no. 35/1999/CPCSEA).

\section{Induction of pulmonary fibrosis}

Briefly, after the weight was recorded, the rats were anesthetized using a combination of ketamin $(80 \mathrm{mg} / \mathrm{kg}$ body weight, i.p.) and xylazine (20 mg/kg body weight, i.p.) as per standard protocol [44]. A midline incision was made in the neck and the exposed trachea was intubated with tracheal cannula under direct visualization. For induction of pulmonary fibrosis, on day 0 the rats received a single dose of $6.5 \mathrm{U} / \mathrm{kg}$ body weights, bleomycin sulfate dissolved in $0.5 \mathrm{~mL}$ of $0.9 \% \mathrm{NaCl}$ solution by intratracheal instillation on day 0 of the experiment [49]. Control rats were given a single intratracheal dose of sterile saline alone.

\section{Experimental groups}

The animals were randomized into 5 groups each consisting of 10 animals [11]:

- $\quad$ Group 1 - Vehicle control rats were given 0.5\% carboxymethylcellulose solution orally from day 1 to day 20 of the experiment.

- Group II - Bleomycin treated rats were treated with $0.5 \%$ carboxymethylcellulose solution orally from day 1 to day 20 of the experiment.

- Group III - Animals were orally administered with quercetin (100 mg/kg body weight/day) in $0.5 \%$ carboxymethylcellulose solution from day 1 to day 20 of the experiment after bleomycin instillation [43].

- $\quad$ Group IV - Animals from were treated with sulindac within its therapeutic anti-inflammatory dose $\left(\mathrm{ED}_{50}\right.$ for rats, 20 $\mathrm{mg} / \mathrm{kg}$ body weight) in $0.5 \%$ methylcellulose solution for day 1 to day 20 of the experiment after bleomycin instillation [45]. 
- Group V - Animals were treated with quercetin $(50 \mathrm{mg} / \mathrm{kg}$ body weight/day) and sulindac (10 $\mathrm{mg} / \mathrm{kg}$ body weight/day) in $0.5 \%$ methylcellulose solution for day 1 to day 20 of the experiment after bleomycin instillation.

The drug was freshly prepared in $0.5 \%$ carboxymethylcellulose solution and the concentration was adjusted so that each animal received $10 \mathrm{ml} / \mathrm{kg}$ body weight. The animals were weighed at the beginning, through and at the end of experiments. The changes in body weight were recorded.

\section{Preparation of lung tissue homogenate}

On day 21 of the experiment, six animals from each group were sacrificed with thiopentone sodium and the lung lobes were excised and weighed. Lungs of four animals from each group were used for histological evaluation.

\section{Collagen specific studies}

Excess collagen deposition is the hallmark of pulmonary fibrosis, which is formed from amino acid precursor, hydroxyproline. Hydroxyproline content present in the lung tissue homogenate of control and experimental groups was performed as described by Edwards and O'brien [7]. After sacrificed, each lung tissue was perfused and fixed in $10 \%$ neutral buffer formalin and routinely processed and embedded in paraffin. Lung tissue collagen content was examined under the light microscopy using Masson trichrome, which is a specific staining for collagen.

\section{Biochemical assays}

Lipid peroxidation was assessed by the measuring the level of malondialdehyde following the method of Ohkawa., et al [28]. The activity of superoxide dismutase was assayed by the method described by Kakkar., et al [18]. The activity of catalase was assessed by Luck [23].

\section{Histological studies}

After sacrificed, each lung tissue was perfused and fixed in $10 \%$ neutral buffer formalin and routinely processed and embedded in paraffin. Serial sections ( $4 \mu \mathrm{m})$ were cut and stained with hematoxylin and eosin for light microscopic elucidate pathological changes associated with bleomycin induced lung fibrosis and efficacy of quercetin and sulindac independently and in combination to examine the degree of fibrosis. The severity of fibrosis was individually accessed using the semi-quantitative grading system described by Szapiel., et al [41]. The scores of fibrosis in lung specimens were graded from - to +++ and correspondingly numbered as from 0 to
3. The entire lung section was reviewed at a magnification of $10 \mathrm{X}$. Each of the 25 random microscopic fields per section were detected, a score ranging from 0 to 3 was assigned. All assessments were performed in blind fashion.

\section{Measurement of tumor necrosis factor- $\alpha$}

Tumor necrosis factor- $\alpha$ in plasma was assayed by specific enzyme-linked immunosorbent assay using commercially available ELISA test kits (XpressBio Life Science Products, USA). The kit contains a tumor necrosis factor- $\alpha$ coat monoclonal antibody to tumor necrosis factor- $\alpha$. There presentative standard curve was generated using the tumor necrosis factor- $\alpha$ standard with the kit.

\section{Materials}

Quercetin, sulindac, chloramine-T and hydroxyproline were procured from Sigma Aldrich Chemie GmbH. Bleomycin hydrochloride was procured from the local market and was in the form of bleomycin ampoules (15 units) manufactured by Biochem Pharmaceutical Industries Ltd., Mumbai, India. All other chemicals were of analytical grade and procured from reputed manufactures of India viz., Sisco Research Laboratories Pvt. Ltd., Qualigens Fine Chemicals Pvt. Ltd. and Himedia Laboratory Pvt. Ltd.

\section{Statistical analysis}

Statistical analyses were carried out by analysis of variance (ANOVA) followed by appropriate post hoc test [multiple comparison tests (LSD)]. All analyses of data were performed using SPSS for windows version 17.0 and a probability value of 0.05 was considered statistically significant.

\section{Results}

\section{Changes in body weight and percent body weight}

Table 1 shows the mean body weight and percent body weight changes in each group. The body weights of all the treatment groups were comparable $(\mathrm{P}<0.05)$ to the control group on days 3 and 7. Single intratracheal administration of bleomycin (6.5 U/ $\mathrm{kg}$ ) resulted in a marked decrease in their body weight on days 14 and 21 as compared $(\mathrm{P}<0.05)$ to the saline treated control group because of severe tissue damage caused by generation of free radicals due to bleomycin instillation. These reductions in bodyweights associated with bleomycin administration were significantly restored by treatment with quercetin or sulindac when compared $(\mathrm{P}$ $<0.05$ ) to the bleomycin treated group. However, no significant difference existed between the two treated groups. Co-administration 
Combined Inhibitory Effect of Sulindac an NSAID and Quercetin an Antioxidant on Bleomycin-induced Lung Fibrosis in Rats

of quercetin and sulindac at the lower doses was associated with significant $(\mathrm{P}<0.05)$ restoration of body weights when compared with the bleomycin treated group and remained comparable to that of the control group rats.

\begin{tabular}{|c|c|c|c|c|c|c|c|c|c|}
\hline \multirow{2}{*}{$\begin{array}{c}\text { Treatment } \\
\text { Groups }\end{array}$} & \multicolumn{4}{|c|}{ Mean Body Weight } & \multicolumn{3}{c|}{ Percent Body Weight Change } \\
\cline { 2 - 11 } & Day 0 & Day 3 & Day 7 & Day 14 & Day 21 & $\begin{array}{c}\text { Day } \\
\mathbf{0}-\mathbf{3}\end{array}$ & $\begin{array}{c}\text { Day } \\
\mathbf{0}-\mathbf{7}\end{array}$ & $\begin{array}{c}\text { Day } \\
\mathbf{0}-\mathbf{1 4}\end{array}$ & $\begin{array}{c}\text { Day } \\
\mathbf{0}-\mathbf{2 1}\end{array}$ \\
\hline & & & & & & & & & \\
Control & 310.40 & 301.70 & 326.70 & 359.80 & 366.00 & -2.80 & 5.22 & 15.88 & 17.88 \\
Group & \pm & \pm & \pm & \pm & \pm & \pm & \pm & \pm & \pm \\
& 17.68 & 17.91 & 22.16 & 27.11 & 25.07 & 1.98 & 2.91 & 4.75 & 3.25 \\
\hline Bleomycin & 320.40 & 303.80 & 314.80 & $320.50^{*}$ & $331.40^{*}$ & $-5.22^{*}$ & $-1.63^{*}$ & $0.27^{*}$ & $3.72^{*}$ \\
Control & \pm & \pm & \pm & \pm & \pm & \pm & \pm & \pm & \pm \\
& 25.36 & 26.72 & 21.21 & 19.31 & 15.50 & 1.99 & 2.89 & 5.05 & 4.75 \\
\hline Quercetin & 318.60 & 300.90 & 308.30 & 338.50 & 348.80 & $-5.59^{*}$ & $-3.25^{*}$ & $6.38^{*} \#$ & $9.59^{*} \#$ \\
Treatment & \pm & \pm & \pm & \pm & \pm & \pm & \pm & \pm & \pm \\
& 26.88 & 27.07 & 28.52 & 23.58 & 25.48 & 1.69 & 2.78 & 2.32 & 2.40 \\
\hline Sulindac & 320.40 & 301.40 & 311.20 & $346.10 \#$ & 359.90 & $-6.00^{*}$ & $-2.87^{*}$ & $8.05^{*} \#$ & $12.37^{*} \#$ \\
Treatment & \pm & \pm & \pm & \pm & \pm & \pm & \pm & \pm & \pm \\
& 24.63 & 27.44 & 25.07 & 27.02 & 27.78 & 2.67 & 2.11 & 3.16 & 3.16 \\
\hline Quercetin + & 318.30 & 302.30 & 316.40 & $349.50 \#$ & 364.20 & $-4.97^{*}$ & $-0.29^{*}$ & $10.30^{*} \#$ & $14.93 \#$ \\
Sulindac & \pm & \pm & \pm & \pm & \pm & \pm & \pm & \pm & \pm \\
Treatment & 23.54 & 21.09 & 23.59 & 24.80 & 26.70 & 2.38 & 8.41 & 10.80 & 11.42 \\
\hline
\end{tabular}

Table 1: Mean body weight and percent body weight changes.

Note: $\mathrm{n}=10$.

Key: ${ }^{*} \mathrm{p}<0.05$ vs. Control group. \# $\mathrm{p}<0.05$ vs. bleomycin group.

A significant $(\mathrm{P}<0.05)$ increase in the percent body weight change was observed in all the three treatment groups on days 14 and 21 of the experiment which was found to be comparable to the bleomycin treated group on days 3 and 7 of the experiment. Moreover, percent body weight change revealed that there was a significant $(\mathrm{P}<0.05)$ decrease in the percent body weight in the entire treatment group throughout the experiment apart from the combination treatment of quercetin and sulindac at the lower dose which was found to be comparable to the control group at the end of the experiment which showed that the combination treatment was found to be more effective than the individual treatment of both the drugs.

\section{Change in percent relative lung weight}

Bleomycin instillation was accompanied by significant $(\mathrm{P}<$ 0.05 ) increase in the percent relative lung weight when compared with the control group as shown in table 2 . This deleterious effect due to treatment of bleomycin instillation was ameliorated by treatment with either quercetin or sulindac compared to bleomycin control group $(\mathrm{P}<0.05)$. Nonetheless, the relative lung weight remained near identical in animals that received the ameliorative agents as individual compound. However, administration of quercetin and sulindac in combination at the lower doses was found more effective.

\begin{tabular}{|c|c|c|c|}
\hline & $\begin{array}{c}\text { Relative Or- } \\
\text { gan Weight }\end{array}$ & $\begin{array}{c}\text { Hydroxypro- } \\
\text { line content }\end{array}$ & $\begin{array}{c}\text { Grade of } \\
\text { fibrosis }\end{array}$ \\
\hline Control Group & $0.63 \pm 0.01$ & $1.71 \pm 0.05$ & $0.00 \pm 0.00$ \\
\hline Bleomycin Control & $0.86 \pm 0.05^{*}$ & $2.59 \pm 0.23^{*}$ & $2.48 \pm 0.07^{*}$ \\
\hline $\begin{array}{c}\text { Quercetin Treat- } \\
\text { ment }\end{array}$ & $0.66 \pm 0.06 \#$ & $1.93 \pm 0.12 \#$ & $1.44 \pm 0.13^{*}, \#$ \\
\hline $\begin{array}{c}\text { Sulindac Treat- } \\
\text { ment }\end{array}$ & $0.70 \pm 0.12 \#$ & $1.94 \pm 0.12^{*}, \#$ & $1.58 \pm 0.15^{*}, \#$ \\
\hline $\begin{array}{c}\text { Quercetin + Sulin- } \\
\text { dac Treatment }\end{array}$ & $0.65 \pm 0.03 \#$ & $1.82 \pm 0.07 \#$ & $0.51 \pm 0.12^{*}, \#$ \\
\hline $\begin{array}{c}\text { Total Number of } \\
\text { Animals in each } \\
\text { Group }\end{array}$ & $\mathrm{N}=6$ & $\mathrm{~N}=6$ & $\mathrm{~N}=4$ \\
\hline \multicolumn{2}{|c|}{} & & \\
\hline
\end{tabular}

Table 2: Relative organ weight, hydroxyproline content and grade of fibrosis.

Key: ${ }^{*} \mathrm{p}<0.05$ vs. Control group. \# $\mathrm{p}<0.05$ vs. bleomycin group. 


\section{Hydroxyproline content}

Mean lung hydroxyproline content in bleomycin treated group and sulindac treated groups were significantly $(\mathrm{P}<0.05)$ higher than that in the control untreated group. Both quercetin and sulindac treated groups exhibited significant $(\mathrm{P}<0.05)$ reduction in hydroxyproline content in comparison with bleomycin control group, with no significant difference between the two treated groups. Co-administration of quercetin and sulindac also displayed a significant $(\mathrm{P}<0.05)$ decline in the higher hydroxyproline content as compared to the sulindac treated group (Table 2).

Figure 1 shows the Masson's trichrome staining. Tissue injury due to administration of bleomycin resulted in the excess deposition of collagen, as evident in figure $1 \mathrm{~B}$ when compared to that of control group (Figure 1A). However, both quercetin and sulindac treated groups exhibited noticeable reduction in the collagen deposition in the lung tissues (Figure $1 \mathrm{C}$ and 1D). Administration of quercetin and sulindac in combination also displayed a noteworthy reduction in the deposition of collagen which can be correlated with the decrease in the hydroxyproline content as shown in table 2 and as observed in figure $1 \mathrm{E}$.
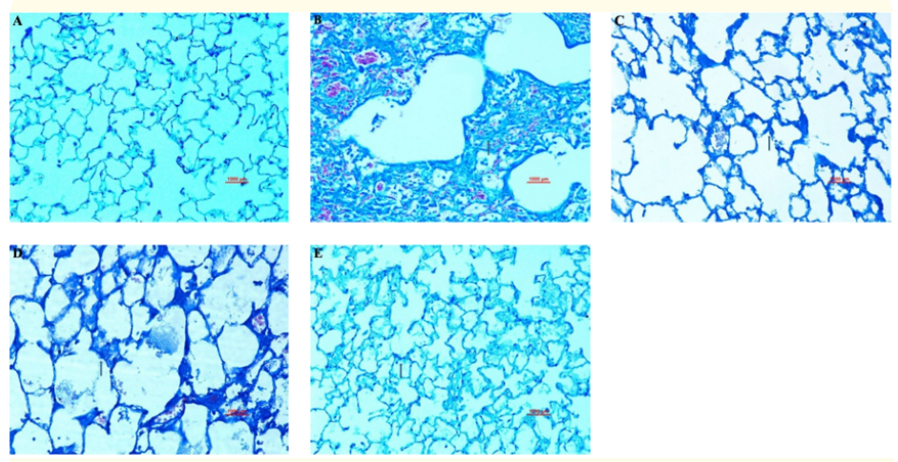

Figure 1: Masson trichrome staining.

A. Vehicle treated lungs showing normal architecture of lung with no evidence of collagen proliferation.

B. Bleomycin treated lung showing severe thickening of alveolar septa with extensive collagen deposition.

C. Quercetin treated lungs showing minimal thickening of alveolar septa with collagen deposition.

D. Sulindac treated lungs showing mild thickening of alveolar septa with collagen deposition.

E. Combined treatment of quercetin sulindac treated showed near normal pulmonary structure with no evidence of collagen deposition.
Biochemical estimations for oxidant stress markers

Bleomycin treatment produced a significant $(\mathrm{P}<0.05)$ increase in the lung tissue MDA content, an index for lipid peroxidation when compared with control groups. As shown in figure 2 bleomycin induced increments in MDA content of the lung were significantly prevented by quercetin and sulindac treatments when compared to that of bleomycin group. Results of the sulindac treatment did show a significant decrease to that of the bleomycin treated group but it still was not comparable to that of vehicle control group at the end of the experiment. However, co-administration of quercetin and sulindac reduced the level of MDA content in the lung tissue and the values were found comparable to that of the control group.

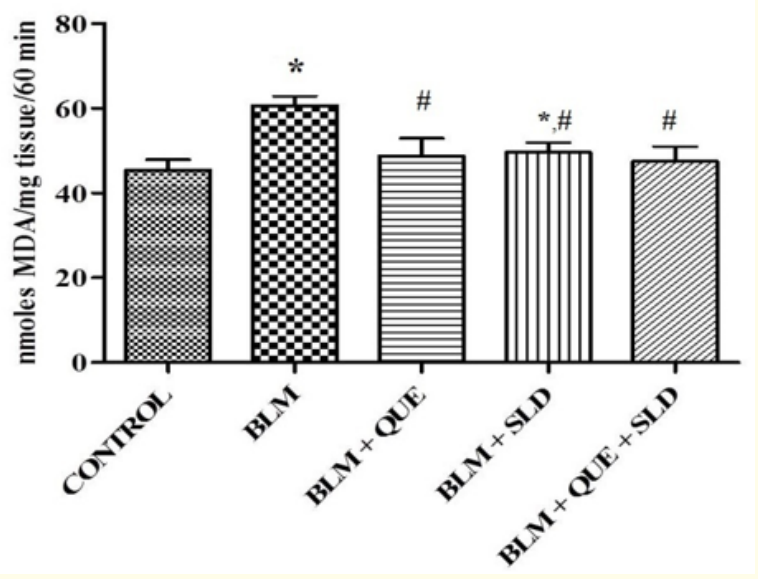

Figure 2: Effect of various treatments on the Malondialdehyde levels in Experimental Groups.

$\mathrm{N}=6 .{ }^{*} \mathrm{p}<0.05$ vs. Control Group. \# $\mathrm{p}<0.05$ vs. bleomycin Group.

Similarly, depletion in the levels of superoxide dismutase and catalase activities in the tissue reflects indirectly the generation of free radical due to bleomycin administration. Intratracheal instillation of bleomycin produced a significant decrease in the superoxide dismutase and catalase activities in lung tissue after 21 days when compared with the control group rats (Figure 3 and 4). Quercetin and sulindac treatments significantly prevented the depletion of superoxide dismutase and catalase activities when compared to that of the bleomycin control group however the results were still significant $(\mathrm{P}<0.05)$ to that of the vehicle control group for both the treatments. Quercetin and sulindac in combination also prevented the decrease in the levels of superoxide dismutase and catalase at the lower doses. 


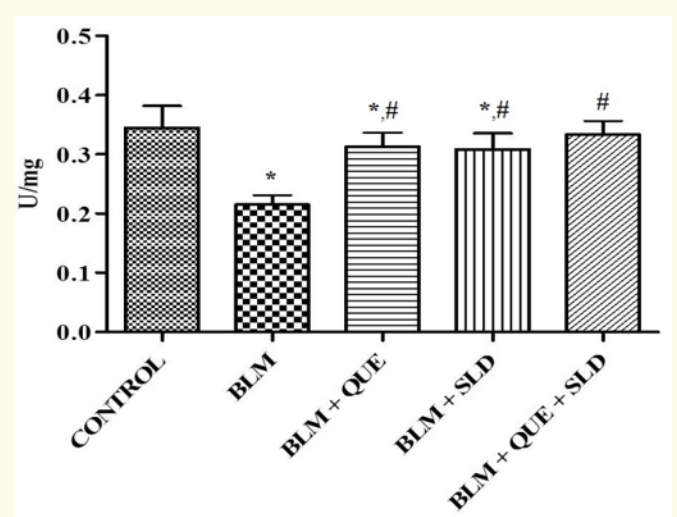

Figure 3: Effect of various treatments on the superoxide dismutase levels in Experimental Groups.

$\mathrm{N}=6 .{ }^{*} \mathrm{p}<0.05$ vs. Control Group. \# $\mathrm{p}<0.05$ vs. bleomycin Group.

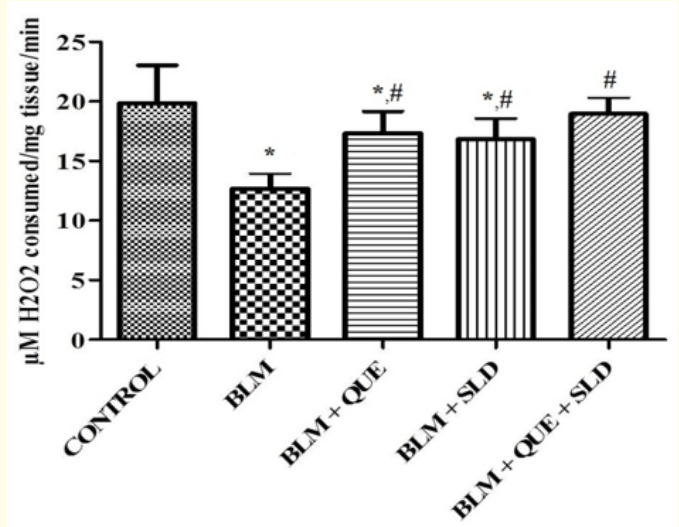

Figure 4: Effect of various treatments on the Catalase levels in Experimental Groups.

$\mathrm{N}=6 .{ }^{*} \mathrm{p}<0.05$ vs. Control Group. \# $\mathrm{p}<0.05$ vs. bleomycin Group.

\section{Tumor necrosis factor- $\alpha$ concentration}

Plasma levels of tumor necrosis factor- $\alpha$ is presented in figure 5 . The tumor necrosis factor- $\alpha$ level in plasma of bleomycin-administered group remained elevated on day 21 when compared with the sham treated group. Treatment with quercetin and sulindac decreased bleomycin induced increases of tumor necrosis factor- $\alpha$ level in plasma at the end of the experiment when compared to bleomycin treated group. However, the levels of tumor necrosis factor- $\alpha$ remained elevated in sulindac treated group on day 21 when compared to that of control group while the levels of tumor necrosis factor- $\alpha$ in quercetin treated group remained comparable to that of the control group on day 21 . Co-administration of quercetin and sulindac was associated with a significant $(\mathrm{P}<0.05)$ reduction in tumor necrosis factor- $\alpha$ concentration in comparison with the sulindac treated group.

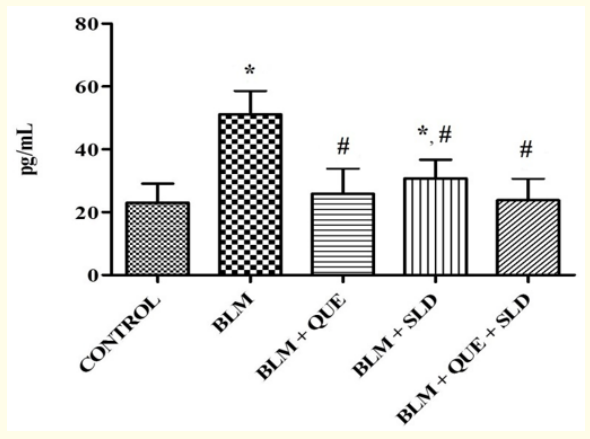

Figure 5: Effect of various treatments on Plasma levels of tumor necrosis factor- $\alpha$ in Experimental Groups.

$\mathrm{N}=10{ }^{*} \mathrm{p}<0.05$ vs. Control Group. \# $\mathrm{p}<0.05$ vs. bleomycin Group.

\section{Histopathological examination of lung tissue}

Histopathological abnormalities in lungs were assessed on day 21 using hematoxylin and eosin staining (Figure 6). Sections from vehicle group displayed normal histoarchitecture with no pathological changes evident (Figure 6A). In bleomycin treated group, marked histopathological changes such as interstitial thickening with inflammatory cell infiltration, extensive collagen deposition, collapsed alveolar spaces were observed (Figure 6B and 6C). Inflammatory infiltrates were localized to the alveolar spaces and peribronchial wall which consisted of mainly fibroblasts, lymphocytes, macrophages and few neutrophils.

Furthermore, there were few foci of alveolar epithelialization and mild hyperplastic bronchial epithelium.

Although fibrotic lesions were observed in quercetin and sulindac treated groups, the extent of alveolitis and fibrosis was markedly less severe compared with that of bleomycin treated group indicated ameliorative effect of these regimens to reduce pulmonary fibrosis (Figure 6D to 6G). Efficacy of quercetin and sulindac to abrogate fibrotic changes when accessed histologically revealed better effect of quercetin than sulindac to reduce inflammatory 


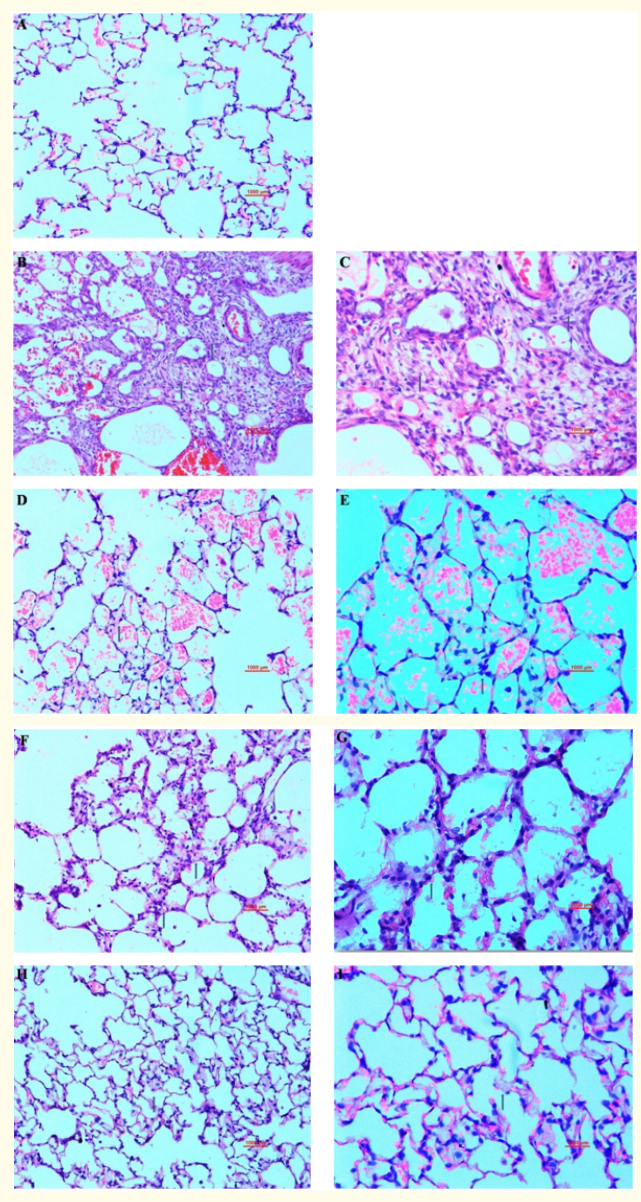

Figure 6: Hematoxylin and Eosin Staining.

A. Control lung showing normal histoarchitecture of lungs - 200X

B. Bleomycin treated lung showing severe thickening of alveolar septa with extensive infiltration of lymphocytes, macrophages, fibroplasia and compressed alveoli - 200X.

C. Bleomycin treated lung showing severe thickening of alveolar septa with extensive infiltration of lymphocytes, macrophages, fibroplasia and collapsed alveoli - 400X.

D. Quercetin treated lung showing minimal septal thickening with mild inflammatory infiltrates and fibrosis - 200X.

E. Quercetin treated lung showing minimal septal thickening with few inflammatory infiltrates - 400X.

F. Sulindac treated lung showing mild thickening of alveolar septa with mononuclear cell infiltration and fibroplasia - 200X

G. Sulindac treated lung showing mild thickening of alveolar septa with mononuclear cell infiltration and fibroplasia - 400X

$\mathrm{H}$. Combined treatment showing near normal histoarchitecture of lung with few mononuclear cell infiltration in alveolar septa - 200X

I. Combined treatment showing near normal histoarchitecture of lung with few mononuclear cell infiltration in alveolar septa - 400X. and fibrotic changes. Lung sections from rats treated with combination therapy of quercetin and sulindac displayed nearly normal alveolar structure except few inflammatory infiltrates in interstitium (Figure 6H and 6I).

Furthermore, the semi-quantitative assessment of fibrosis in lung sections was performed by scoring pathological lesions as per the Szapiel method of examination (Table 2). The Szapiel score of bleomycin induced group was found significantly higher on day 21 when compared with control group. However, Szapiel scores on day 21 of quercetin and sulindac treated groups showed marked decrease compared to the bleomycin treated group reaffirming ameliorative role of quercetin and sulindac against bleomycin induced lung fibrosis. Scores for the combination therapy of quercetin and sulindac displayed significant improvement and was found to be near normal (Table 2).

\section{Discussion}

Idiopathic pulmonary fibrosis is an end-stage pulmonary disease for which efficient treatment is not available. The disease is characterized by failure of alveolar re-epithelialization, deposition of excessive collagen and distortion of the lung architecture, which leads to respiratory failure [29]. To understand the finer mechanisms of development of pulmonary fibrosis as well as to screen the efficacy of various compounds as potential therapeutic agent against this pathological manifestation animal experimentations are inevitable. The bleomycin model of lung fibrosis is widely used and is one of the standardized experimental models for human lung fibrosis [20,39]. Comparison studies of patients with lung pneumopathy and experimentally induced lung fibrosis animal model have validated effectiveness of this experimental system as an acceptable model. Subsequently, the use of this animal model has helped in partly establishing the pathways of lung damage leading to fibrosis [5].

In the current study we have used Wistar rat model of lung fibrosis created by challenging the rats with a single dose of bleomycin sulfate by intratracheal instillation. A significant decrease in the body weight and an increase in the relative organ weight of lungs were observed in the bleomycin treated group, which could be attributed to the progression of the fibrosis [38,54]. The present study also revealed that bleomycin instillation lead to an abnormal increase in hydroxyproline level, an increase in the amount of collagen deposition along destruction of the lung architecture such as collapse of alveolar spaces, thickened alveolar wall and infiltration of inflammatory cells such as macrophages, neutrophils and lymphocytes which play a key role in inflammation and tis- 
sue remodelling and is in accordance with the previous findings $[19,38,47,52,53]$.

It is well documented that free radical target biomacromolecules such as DNA, protein and lipid, which lead to lipid peroxidation, resulting in damage to the lung [22]. In the current study, single intra tracheal instillation of bleomycin lead to an exemplified heightened MDA activity in the lungs. Moreover, the bleomycin treated rats also showed compromised antioxidant response as apparent from subdued activity of antioxidant enzymes such as catalase and superoxide dismutase. Similar observations made by others give credence to the present notion [14,37]. Moreover, it is reported that TNF- $\alpha$, a potent pro-inflammatory cytokine, acts as a major component of a multifaceted network of cellular and molecular interactions that regulate the progression of fibrotic process [32,34]. In this study, a significant elevation in the expression of TNF- $\alpha$ was observed in the bleomycin treated group, which is in accordance with previous findings $[48,51]$.

Further, it is well known that reactive oxygen species play a critical role in the development of fibrotic in responses to bleomycin challenge. Bleomycin binds to iron (Fe II), undergoes redox cycling and catalyzes the formation of reactive oxygen species $[27,40]$. Flavonoids are reported to be powerful antioxidants providing remarkable protection against oxidative stress and free radical damage. Moreover, quercetin, a member of the flavonoid family, is cited as one of the most prominent dietary antioxidants [4]. In line with this report and the results of the present study revealed that repeated administration of this flavonoid resulted in marked increase in the body weight as compared to the bleomycin treated group and the relative organ weight of lungs remained comparable to the control group rats.

Moreover, repeated administrations of quercetin also lead to significant decrease in the hydroxyproline level which was also conformed visually by masson's trichrome staining of lung sections. The results of the biochemical estimations for the markers of the oxidative stress also showed that the repeated treatment of quercetin significantly $(\mathrm{P}<0.05)$ inhibited the production of the reactive oxygen species produced by the administration of bleomycin which can primarily be credited to presence of the phenolic hydroxyl groups in the structure of quercetin [25].

In the present study it was also observed that quercetin treatment decreased the progression of bleomycin induced structural deformation as exemplified by the low Szapiel scores in this group of animals compared to bleomycin treated ones. A decrease in the level of TNF- $\alpha$ in the plasma was also observed. Oxidative status leads to the activation of the MAPK pathways which is a known target for potential fibrosis therapy, as several fibrogenic cytokines signal through MEK/ERK, including noncanonical TGF- $\beta$, PDGF, IL13 and TNF- $\alpha[17,21,24]$. Therefore, quercetin, due to its antioxidative property and inhibitory effect on TNF- $\alpha$, targets the abovementioned pathway which may ultimately lead to the ameliorative effect of quercetin in Wistar rats.

In the present study, it appeared that anti-inflammatory of sulindac reduced the severity of body weight loss, with suppression on the increase in the relative organ weight that accompanied bleomycin instillation as it is well known that inflammation does play an important role in the development of fibrosis in response to bleomycin instillation [52]. Moreover, it was also observed that the increase in the hydroxyproline and collagen deposition in the tissue was also inhibited by the repeated treatment of this NSAID in comparison to that of the bleomycin instillation $(\mathrm{P}<0.05)$. Moreover, the biochemical estimations of the markers for the oxidative stress revealed that the repeated oral administration of this NSAID significantly $(\mathrm{P}<0.05)$ reduced the production of the reactive oxygen species due to the administration of bleomycin.

In the present study it was also observed that the repeated treatment of sulindac also improved histological architecture and improved the pathological grading of lung fibrosis. A significant decrease in the level of TNF- $\alpha$ in the plasma was also observed in the sulindac treated rats as compared to that of the bleomycin treated rats which may be attributed to the anti-inflammatory of sulindac. It has been reported that the tissue injury caused by bleomycin is inflammation mediated which might be due to the production of free radicals which possibly leads to activation of NF- $\kappa B$ and increase in the synthesis of tumor necrosis factor- $\alpha[8,19,30]$. Sulindac has an inhibitory effect on NF- $\kappa$ B activity $[3,33]$. Therefore, we suggest that as sulindac reduced the expression of tumor necrosis factor- $\alpha$, it may have an inhibitory effect on NF- $\kappa B$ activity which may be one of the possible mechanisms of the ameliorative effect of sulindac in wistar rats against bleomycin challenged rats.

The result of the present study also suggests that each of quercetin and sulindac exerted a significant attenuation of the extent and severity of bleomycin induced lung fibrosis almost equally; however, the antioxidant was found to be slightly better over the 
NSAID. Additionally, it could also be presumed that attenuating effect of either quercetin or sulindac is partly due to their inhibitory effect on hydroxyproline contents and tumor necrosis factor- $\alpha$. These ameliorative effects associated with quercetin and sulindac treatments could be due to the action of both the drugs on the inflammatory phase of bleomycin challenge.

Moreover, the present study also demonstrated that co-administration of quercetin and sulindac at the low doses appeared to be statistically more benefits than the full separate doses of each on the detrimental effects accompanied bleomycin administration studied in the present study. It may be due to the anti-inflammatory effect of sulindac which is being potentiated by the antioxidant effect of quercetin.

\section{Conclusion}

In conclusion, the present study provided an indication of the possible use of anti-inflammatory and antioxidant an in-attenuating bleomycin induced lung fibrosis by decreasing hydroxyproline level and down regulation of tumor necrosis factor- $\alpha$ mediated collagen deposition in bleomycin challenged Wistar rats. Also, the co-administration of quercetin and sulindac enhanced the beneficial effects afforded by either quercetin or sulindac. This may open whole new perspective for possible roles of these drugs as a target in prevent bleomycin-induced lung damage.

\section{Conflict of Interest Statement}

The authors declare that there are no conflicts of interest.

\section{Acknowledgments}

The authors are very grateful to Dr A. Deshpande for their kind support in providing the facility to conduct the study at the experimental premises. This work was financially supported by Jai Research Foundation, Vapi, India. We thank the anonymous reviewers for their constructive comments that helped to improve the manuscript.

\section{Bibliography}

1. Atzori L., et al. "Attenuation of bleomycin induced pulmonary fibrosis in mice using the heme oxygenase inhibitor Zn-deuteroporphyrin IX-2,4-bisethylene glycol". Thorax 59 (2004): 217-223.

2. Beigh S., et al. "Bleomycin-induced pulmonary toxicopathological changes in rats and its prevention by walnut extract". Biomedicine and Pharmacotherapy 94 (2017): 418-429.
3. Berman K., et al. "Sulindac Enhances Tumor Necrosis Factor$\alpha$-mediated Apoptosis of Lung Cancer Cell Lines by Inhibition of Nuclear Factor-кB". Clinical Cancer Research 8 (2002): 354360.

4. Boots AW., et al. "Health effects of quercetin: from antioxidant to nutraceutical". European Journal of Pharmacology 585 (2008): 325-337.

5. Cooper Jr JAD. "Pulmonary fibrosis". American Journal of Respiratory Cell and Molecular Biology 22 (2000): 520-523.

6. Dharani M and Kalava S. "Ameliorative effect of Punica granatum L. Against bleomycin induced pulmonary fibrosis in rats". International Journal of Pharmaceutical Sciences and Research 6.4 (2015): 1465-1472.

7. Edwards CA., et al. "Modified Assay for Determination of Hydroxyproline in a Tissue Hydrolyzate". Clinica Chimica Acta 104 (1980): 161-167.

8. El-Khoulya D., et al. "Thymoquinone blocks lung injury and fibrosis by attenuating bleomycin-induced oxidative stress and activation of nuclear factor Kappa-B in rats". Toxicology 302 (2012): 106-113.

9. Eynott PR., et al. "Role of nitric oxide in allergic inflammation and bronchial hyperresponsiveness". European Journal of Pharmacology 452 (2002): 123-133.

10. Filderman AE., et al. "Alteration in pulmonary protective enzymes following systemic bleomycin treatment in mice". Biochemistry and Pharmacology 37 (1988): 1111-1116.

11. Gad SC., et al. "Statistics for Toxicology, third ed". In Hayes, A.W. (Eds), Principles and Methods of Toxicology, Raven Press Ltd., New York (1994): 221-274.

12. Galvan L., et al. "Inhibition of bleomycin-induced DNA breakage by superoxide dismutase". Cancer Research 41 (1981): 5103-5106.

13. Gao J., et al. "Antifibrosis effects of total glucosides of DangguiBuxue-Tang in a rat model of bleomycin-induced pulmonary fibrosis". Journal of Ethnopharmacology 136 (2011): 21-26.

14. Grande NR., et al. "Lung fibrosis induced by bleomycin: structural changes and overview of recent advances". Scanning Microscope 12 (1998): 487-494.

15. Gross TJ and Hunninghake GW. "Idiopathic pulmonary fibrosis". The New England Journal of Medicine 345.16 (2001): 517525. 
16. Hagiwara SI., et al. "Aerosolized administration of N-acetyl cysteine attenuates lung fibrosis induced by bleomycin in mice". American Journal of Respiratory and Critical Care Medicine 162 (2000): 225-231.

17. Ho BY., et al. "Dimerumic Acid Inhibits SW620 Cell Invasion by Attenuating H2O2-Mediated MMP-7 Expression via JNK/C-Jun and ERK/C-Fos Activation in an AP-1-Dependent Manner". International Journal of Biological Sciences 7.6 (2011): 869-880.

18. Kakkar P., et al. "A Modified spectrophotometric assay of superoxide dismutase". Indian Journal of Biochemistry and Biophysics 21 (1984): 130-132.

19. Kalayarasan S., et al. "Diallyl sulfide attenuates bleomycin- induced pulmonary fibrosis: Critical role of iNOS, NF- $\mathrm{KB}, \mathrm{TNF}-\alpha$ and IL-1ß". Life Sciences 82 (2008): 1142-1153.

20. Keane MP., et al. "IL-12 attenuates bleomycin induced pulmonary fibrosis". American Journal of Physiology - Lung Cellular and Molecular Physiology 281 (2001): 92-97.

21. Liang S., et al. "The role of NADPH oxidases (NOXs) in liver fibrosis and the activation of myofibroblasts". Frontiers in Physiology 7 (2016): 17.

22. Liang X., et al. "Effect of Feining on bleomycin-induced pulmonary injuries in rats". Journal of Ethnopharmacology 134 (2011): 971-976.

23. Luck H. "A spectrophotometric method for the estimation of catalase". in: Bergmeyer, H.U., (Eds.), Methods of enzymatic analysis. Academic Press, New York (1963): 886-887.

24. Madala SK., et al. "MEK-ERK Pathway Modulation Ameliorates Pulmonary Fibrosis Associated with Epidermal Growth Factor Receptor Activation". American Journal of Respiratory Cell and Molecular Biology 46 (2012): 380-388.

25. Materska M and Perucka I. "Antioxidant Activity of the Main Phenolic Compounds Isolated from Hot Pepper Fruit (Capsicum annuum L.)". Journal of Agricultural and Food Chemistry 53 (2005): 1750-1756.

26. Moeller A., et al. "The bleomycin animal model: A useful tool to investigate treatment options for idiopathic pulmonary fibrosis?" The International Journal of Biochemistry and Cell Biology 40 (2008): 362-382.

27. Myers JM., et al. "Redox activation of Fe (III)-thiosemicarbazones and Fe (III)-bleomycin by thioredoxin reductase: speci- ficity of enzymatic redox centers and analysis of reactive species formation by ESR spin trapping". Free Radical Biology and Medicine 60 (2013): 183-194.

28. Ohkawa H., et al. "Assay of lipoperoxides in animal tissues by thiobarbituric acid reaction". Analytical Biochemistry 95 (1979): 351-358.

29. Oku H., et al. "Antifibrotic action of pirfenidone and prednisolone: Different effects on pulmonary cytokines and growth factors in bleomycin-induced murine pulmonary fibrosis". European Journal of Pharmacology 590 (2008): 400-408.

30. Ortiz LA., et al. "Enalapril protects mice from pulmonary hypertension by inhibiting TNF-mediated activation of NF-kB and AP-1". American Journal of Physiology - Lung Cellular and Molecular Physiology 282 (2002): 1209-1221.

31. Oury TD., et al. "Attenuation of bleomycin-induced pulmonary fibrosis by a catalytic antioxidant metalloporphyrin". American Journal of Respiratory Cell and Molecular Biology 25 (2001): 164-169.

32. Pallai A., et al. "Transmembrane TNF- $\alpha$ Reverse Signaling Inhibits Lipopolysaccharide-Induced Proinflammatory Cytokine Formation in Macrophages by Inducing TGF- $\beta$ : Therapeutic Implications". The Journal of Immunology 196.3 (2016): 11461157.

33. Park MH and Hong JT. "Roles of NF- $\mathrm{BB}$ in cancer and inflammatory diseases and their therapeutic approaches". Cells 5 (2016): 15.

34. Razzaque MS and Taguchi T. "Pulmonary fibrosis: cellular and molecular events". Pathology International 53 (2003): 133145.

35. Reyfman PA., et al. "Transcriptional profiling of sorted alveolar macrophages reveals homology between human fibrotic interstitial lung disease and an animal model of pulmonary fibrosis. In B97. Flipping the Switch: Determinants of Fibrosis". American Thoracic Society (2017): A4667-A4667.

36. Rocca J., et al. "New use for an old drug: COX-independent antiinflammatory effects of sulindac in models of cystic fibrosis". British Journal of Pharmacology 173.11 (2016): 1728-1741.

37. Sogut S., et al. "Erdosteine prevents belomycin-induced pulmonary fibrosis in rats". European Journal of Pharmacology 494 (2004): 213-220. 
38. Soumyakrishnan S and Sudhandiran G. "Daidzein attenuates inflammation and exhibits antifibrotic effect against bleomycin-induced pulmonary fibrosis in Wistar rats". Biomedicine and Preventive Nutrition 1 (2011): 236-244.

39. Spagnolo P., et al. "Idiopathic pulmonary fibrosis: recent advances on pharmacological therapy". Pharmacology and Therapeutics 152 (2015): 18-27.

40. Sriram N., et al. "Epigallocatechin-3-gallate augments antioxidant activities and inhibits inflammation during bleomycininduced experimental pulmonary fibrosis through Nrf2-Keap1 signaling". Pulmonary Pharmacology and Therapeutics 22 (2009): 221-236.

41. Szapiel SV., et al. "Bleomycin -induced interstitial pulmonary disease in the nude, athymic mouse". American Review of Respiratory Diseases 120 (1979): 893-899.

42. Tajima S., et al. "Effects of edaravone, a free-radical scavenger, on bleomycin-induced lung injury in mice". European Respiratory Journal 32.5 (2008): 1337-1343.

43. Tang Y., et al. "Quercetin prevents ethanol-induced dyslipidemia and mitochondrial oxidative damage". Food and Chemical Toxicology 50 (2012): 1194-1200.

44. Teixeira KC., et al. "Attenuation of bleomycin-induced lung injury and oxidative stress by $\mathrm{N}$-acetylcysteine plus deferoxamine". Pulmonary Pharmacology and Therapeutics 21 (2008): 309-316.

45. Vaish V., et al. "Role of Sulindac and Celecoxib in the regulation of angiogenesis during the early neoplasm of colon: Exploring PI3-K/PTEN/Akt pathway to the canonical Wnt/b-catenin signaling". Biomedicine and Pharmacotherapy 66 (2012): 354367.

46. Vane JR., et al. "Cyclooxygenases 1 and 2". Annual Review of Pharmacology and Toxicology 38 (1998): 97-120.

47. Varol C., et al. "Macrophages: development and tissue specialization”. Annual Review of Immunology 33 (2015): 643-675.

48. Verma R., et al. "Evaluating the inhibitory potential of sulindac against the bleomycin-induced pulmonary fibrosis in wistar rats". Environmental Toxicology and Pharmacology 36 (2013): 769-778.

49. Wang HD., et al. "Bilirubin ameliorates bleomycin-induced pulmonary fibrosis in rats". American Journal of Respiratory and Critical Care Medicine 165 (2002): 406-411.
50. Wang QJ., et al. "Amelioration of bleomycin-induced pulmonary fibrosis in hamsters by combined treatment with taurine and niacin". Biochemistry and Pharmacology 42 (1991): 11151122.

51. Weissler JC. "Idiopathic pulmonary fibrosis: cellular and molecular pathogenesis". American Journal of the Medical Sciences 297 (1989): 92-104.

52. Xin Wei X., et al. "A phosphoinositide 3-kinase- $\gamma$ inhibitor, AS605240 prevents bleomycin-induced pulmonary fibrosis in rats". Biochemical and Biophysical Research Communications 397 (2010): 311-317.

53. Zhao L., et al. "Neferine, a bisbenzylisoquinline alkaloid attenuates bleomycin induced pulmonary fibrosis". European Journal of Pharmacology 627 (2010): 304-312.

54. Zhou X., et al. "Inhibitory effects of Hu-qi-yin on the bleomycin-induced pulmonary fibrosis in rats". Journal of Ethnopharmacology 111 (2007): 255-264.

\section{Volume 4 Issue 9 September 2021}

(C) All rights are reserved by Suresh Balakrishnan., et al. 